\title{
A NOTE ON FLAT MODULES OVER F-ALGEIBRAS
}

\author{
BORIS LAVRIČ
}

\begin{abstract}
Let $A$ be an Archimedean uniformlly complete unital f $f$-algebra. It is proved that the following conditions are equivalent: (1) $A$ is a Bezout ring; (2) $A$ is a $P F$-ring; (3) Every ideal of $A$ is flat; (4) Every submodule of a free $A$-module is that. This extends a result by $C$. Neville on algebras of type $C(\mathbb{X})$.
\end{abstract}

\section{Introduction}

Let $X$ be completely regular Hausdorff topological space, and let $C(X)$ be the ring of all continuous real-valued functions on $X$. Using the well known topological characterization of spaces $X$ for which $C(X)$ is a Bezout ring [5] C. Neville has proved in [7] that the following conditions are equivalent.

(1) $C(X)$ is a Bezout ring.

(2) $C(X)$ is a $\mathbb{P F}$-ring.

(3) Every ideal of $C(X)$ is flat.

(4) Every submodule of a free $C(X)$-module is flat.

It is the aim of this note to extend this result to uniformly complete Archimedean unital $f$-algebras.

For the terminology and general theory of rings and modules we refer the reader to [1], [4], and for elementary $f$-algebra theory we refer to [6] and [8].

All rings considered in the paper are assumed to be commutative and with unit element. A ring $R$ is called a Bezout ring whenever every finitely generated ideal of $\mathbb{R}$ is principal. A ring $\mathbb{R}$ is called a $P F$-ring if every principal ideal of $\mathbb{R}$ is a flat $\mathbb{R}$-module. A ring $R$ is said to be reduced (or semiprime) if it has no nonzero nilpotent elements.

A lattice ordered real algebra $A$ is called an $f$-algebro whenever

$$
a \wedge b=0, a, b \in A \text { implies } a c \wedge b=c a \wedge b=0
$$

Receive March 25, 1991.

1985 Mathematics Subject Classification. 13C11, 06F25

Key words and phrases: Archimedean $f$-algebra, Bezout ring, PF-ring, flat module.

This work was supported by the Research Council of Slovenia. 
for all $c \in A^{+}$. It is well known that an Archimedean $f$-algebra $A$ with unit is commutative, reduced, and that $a, b \in A$ satisfy $a b=0$ if and only if $|a| \wedge|b|=0$. An $f$-algebra $A$ is said to be normal if

$$
a, b \in A^{+}, a \wedge b=0 \text { implies }\{a\}^{d}+\{b\}^{d}=A,
$$

where $G^{d}=\{h \in A:|g| \wedge|h|=0$ forall $g \in G\}$ for $G \subset A$. It follows that an Archimedean unital $f$-algebra $A$ is normal if and only if

$$
a b=0, a, b \in A \text { implies } a n n(a)+a n n(b)=A
$$

\section{Results}

First we state a criterion characterizing those principal ideals of a. ring $R$ which are flat $R$-modules. Since the criterion is an easy consequence of $[2, \mathbb{P r o p}$. 2.3] or $[1,1.2 .11$. Cor.1], we omit its proof.

Lemma 1. A principal ideal (a) of a ring $\mathbb{R}$ is a flat $\mathbb{R}$-module if and only if for each $b \in R$ satisfying $a b=0$ there exist elements $a_{1}, a_{2}, \ldots, a_{n} \in(a)$ and $b_{1}, b_{2}, \ldots, b_{n} \in \mathbb{R}$ such that

$$
a=\sum_{i=1}^{n} a_{i} b_{i} \text { and } b_{i} b=0, i=1,2, \cdots, n \text {. }
$$

Proposition 1 . For a ring $R$ the following conditions are equivalent.

(i) $\mathbb{R}$ is a $\mathbb{P} F$-ring.

(ii) $a, b \in \mathbb{R}, a b=0$ implies that $a n n(a)+a n n(b)=R$.

Proof. (i) $\Rightarrow$ (ii). Let $a, b \in \mathbb{R}$ satisfy $a b=0$. If $R$ is a PF-ring, then $(a)$ is flat, hence by Lemma 1 we have

$$
a=\sum_{i=1}^{n} a_{i} b_{i} \text { and } b_{i} b=0, i=1,2, \cdots, n
$$

for some $a_{1}, \ldots, a_{n} \in(a), b_{1}, \ldots, b_{n} \in R$. Write

$$
a_{i}=c_{i} a, c_{i} \in R, i=1,2 \cdots, n \text {. }
$$

It follows that $a=\left(\sum_{i=1}^{n} b_{i} c_{i}\right) a$, and therefore

$$
1-\sum_{i=1}^{n} b_{i} c_{i} \in \operatorname{ann}(a), \sum_{i=1}^{n} b_{i} c_{i} \in \operatorname{ann}(b),
$$


so (ii) follows.

(ii) $\Longrightarrow$ (i). We shall prove that ( $a)$ is fllat for each $a \in \mathbb{R}$. Suppose that $b \in R$ satisfies $a b=0$. By (ii) there exist $c \in \operatorname{ann}(a)$ and $d \in \operatorname{ann}(b)$ with $c+d=1$. So

$$
a=a 1=a c+a d=a d \text { and } d b=0,
$$

thus by Lemma $1(a)$ is flat.

Corollary. Let $A$ be an Archimedean unital f-algebra. Then $A$ is a PF-ring if and only if $A$ is normal.

Since by $[6] C(X)$ is normal if and only if $X$ is an $F$-space, the above corollary generalizes [7, Cor.1.7].

Lemma 2. Let $\mathbb{R}$ be a reduced Bezout ring. Then every ideal of $\mathbb{R}$ is flat.

Proof. Since a module is fiat if and only if every finitely generated submodule is flat, and since $R$ is Bezout, it suffices to show that $R$ is a $P F$-ring.

Now for any $a, b \in R$ with $a b=0$, we shall check that $\operatorname{ann}(a)+\operatorname{ann}(b)=R$.

Since $(a, b)=(c)$ for some $c \in R$, we have

$$
a=a_{1} c, b=b_{1} c, c=a_{2} a+b_{2} b
$$

for some $a_{1}, b_{1}, a_{2}, b_{2} \in R$. Since $R$ is reduced,

$$
\left(a_{1} b_{1} c\right)^{2}=a_{1} b_{1}(a b)=0
$$

implies $a_{1} b_{1} c=0$. It follows that $d=a_{1} a_{2}$ satisfies

$$
b d=b_{1} c a_{1} a_{2}=0
$$

and

Hence

$$
\begin{aligned}
a(1-d) & =a-a a_{1} a_{2}=a_{1} c-a a_{1} a_{2} \\
& =a_{1}\left(a_{2} a+b_{2} b\right)-a a_{1} a_{2}=a_{1} b_{1} c b_{2}=0 .
\end{aligned}
$$

$$
1=(1-d)+d \in \operatorname{ann}(a)+\operatorname{ann}(b)
$$

and therefore $a n n(a)+a n n(b)=R$.

We are now in a position to extend a part of theorem 3.1 from [7] on modules over reduced Bezout rings. A short proof suggested by the referee is based on [3]. For the sake of completeness we shall repeat briefly the arguments used in the proof of [3, V.Lemma $6.8]$.

Proposition 2. Let $R$ be a reduced Bezout ring. Then every submodule of a free $R$-module is flat. 
Proof. Let $F$ be a free module with base $X$, and let $M$ be its submodule. Assuming the axiom of choice wre may suppose the elements of $X$ are well ordered: $\mathfrak{x}_{1}, \mathfrak{x}_{2}, \ldots, \mathfrak{x}_{\alpha}, \ldots$. For each ordinal $\alpha$, let $M_{\alpha}$ be the submodule of those elements of $M$ which are linear combinations of the $x_{\beta}$ with $\beta \leq \alpha$, and let $N_{\alpha}$ be the submodule of all linear combinations of the $x_{\beta}$ with $\beta<\alpha$.

Each $m \in M_{\alpha}$ is decomposed uniquely into

$$
m=n+r(m) x_{\alpha}, n \in N_{\alpha}, r(m) \in \mathbb{R},
$$

so $M_{\alpha}$ is a direct sum of $N_{\alpha t}$ and $\mathbb{K}_{\alpha}=r\left(M_{\alpha}\right) x_{\alpha}$. Observe that $\mathbb{I}_{\alpha t}=r\left(M_{\alpha}\right)$ is an ideal of $\mathbb{R}$, and that $M$ is a direct sum of all $\mathbb{K}_{\alpha}$ [3]. Since by Lemma 2 each $\mathbb{K}_{\alpha}$ is flat, it follows by [1, 1.2. Prop.2] that $M$ is flat.

We are prepared to prove the main result of the present note.

Theorem. Let $A$ be an Archimedean uniformly complete unital f-algebra. Then the following conditions are equivalent.

(i) $A$ is a Bezout ring.

(ii) $A$ is a $\mathbb{P} F$-ring.

(iii) Every ideal of $A$ is flat.

(iv) Every submodule of a free A-module is flat.

Proof. The implication (i) $\Longrightarrow$ (iv) follows by Proposition 2, while (iv) $\Longrightarrow$ (iii) and (iii) $\Longrightarrow$ (ii) are obvious. To prove (ii) $\Longrightarrow$ (i) suppose that $A$ is a PF-ring. Then by Corollary $A$ is normal, hence Bezout by [6, Theorem 6.6].

Some other characterizations of an $f$-algebra satisfying the conditions of the above theorem are contained in [6], where also the following example is given. It shows that the uniform completeness cannot be dropped from the Theorem.

Example. Let $A$ be the $f$-algebra of all real functions $f$ on the aquare $E=$ $[0,1] \times[0,1]$ for which there exist disjoint sets $\mathbb{E}_{1}, \ldots, \mathbb{E}_{n(\rho)}$ such that $\mathbb{E}=\mathbb{E}_{1} \cup \ldots \mathbb{E}_{n(\rho)}$,
and polynomials $p_{k} \in \mathbb{R}[X, Y]$ satisfing

$$
\left.f\right|_{E_{k}}=p_{l k}, k+1, \ldots, n(f) .
$$

Clearly $A$ is an Archimedean unital $f$-algebra. By [6] $A$ is normal, hence a $\mathbb{P F}$-ring, although $A$ is not a Bezout ring [6]. We claim that $A$ does not satisfy the condition (iii)
of the Theorem.

Let $I$ be the ideal generated by elements

$$
f, g \in A, f(x, y)=x, g(x, y)=y .
$$

We will show that $I$ is not flat. Consider the exact sequence

$$
0 \longrightarrow K \longrightarrow A \oplus A \stackrel{\varphi}{\longrightarrow} I \longrightarrow 0,
$$


where $\varphi$ is defined by $\varphi(a, b)=a f+b g$, and suppose that I is flat. Then by [2, Proposition 2.2] or by $[4,11.27]$ there exists a homomorphism $\psi: A \oplus A \rightarrow \mathbb{K}$ such that $\psi(g,-f)=$ $(g,-f)$. If

$$
\psi(\mathbb{1}, 0)=\left(a_{1} b_{1}\right), \psi(0,1)=\left(a_{2}, b_{2}\right)
$$

then

$$
\begin{gathered}
a_{1} f+b_{1} g=a_{2} f+b_{2} g=0, \\
g=a_{1} g-a_{2} f,-f=b_{1} g-b_{2} f .
\end{gathered}
$$

Using the fact that these equalities cannot hold in $R[X, Y]$ it is not difficult to see that they are contradictive also in $A$, so $I$ is not flat.

\section{References}

[1] N. Bourbalki, Commutative algebra, Addison-Wesley, Reading, 1972.

[2] S. U. Chase, "Direct products of modules," Trns. Amer. Math. Soc. 97 (1960), 457-473.

[3] S. Eilenberg, N. Steenrod, Foundations of algebraic topology, Princeton Univ. Press, Princeton, New Yersey, 1952.

[4] C. Faith, Algebra: rings, modules and categories I, Springer-Verlag, Berlin, Heidelberg, New Yorls, 1973.

[5] L. Gillman, M. Henriksen, "Rings of continuous functions in which every finitely generabed ideal is principal," Trans. Amer. Math. Soc. 82 (1956), 366-391.

[6] C. B. Huijsmans, B. de Pagter, "Ideal theory in f-algebras," Trens. Amer. Math. Soc. 269 (1982), 225-245.

[7] C. W. Neville, "Flat $C(X)$-modules and F-spaces," Math. Proc. Camb. Phil. Soc. 106 (1989), 237-244.

[8] A. C. Zaanem, Riesz spaces II, North-Holland, Amsterdam, 1983.

Institute of Mathematics, Physics and Mechanics, Jadranslea 19, 61000 Ljubljana, Yugoslavia. 